\title{
Magnetoresistance in triphenyl-diamine derivative blue organic light emitting devices
}

\author{
P. Shakya, P. Desai, T. Kreouzis, and W. P. Gillin ${ }^{\text {a) }}$ \\ Department of Physics, Queen Mary, University of London, Mile End Road, London E1 4NS, \\ United Kingdom
}

(Received 15 October 2007; accepted 19 December 2007; published online 20 February 2008)

\begin{abstract}
Magnetoresistance measurements have been performed on thin layers of the triphenyl-diamine derivative, $N, N^{\prime}$-diphenyl- $N, N^{\prime}$ bis(3-methylphenyl)-(1,1'-biphenyl)-4, $4^{\prime}$ diamine (TPD). At low drive voltages, where the current transport is solely hole mediated, no magnetoresistance is observed. At higher drive voltages, where electron injection into the TPD is occurring, magnetoresistance is seen and the sign of the magnetoresistance depends on the current density in the device. (C) 2008 American Institute of Physics. [DOI: 10.1063/1.2885097]
\end{abstract}

\section{INTRODUCTION}

Organic magnetoresistance (OMR) was first observed in organic light emitting diode (OLED) structure by Kalinowski et al. ${ }^{1}$ in 2003. They found that in an aluminum tris $(8-$ hydroxyquinolinate) $\left(\mathrm{Alq}_{3}\right)$ based OLED both the light output and the current through the device were modulated by the presence of an applied magnetic field. The effect of the applied field on the light output was attributed to the hyperfine scale mixing of the singlet and triplet states ${ }^{2,3}$ resulting in an increased singlet concentration and hence greater efficiency. The effect of the field on the current through the device was attributed to this increase in singlet exciton concentration affecting the dissociation current in the device. The effect has been subsequently been studied in a number of other systems including a range of polymers such as poly(9,9-dioctylfluorenyl-2,7-diyl) (Ref. 4) (PFO) and polythiophenes, ${ }^{5}$ as well as small molecules such as pentacene, ${ }^{5} \mathrm{Alq}_{3},{ }^{1,5-7}$ and tris(2-phenylpyridine) iridium. ${ }^{6,8}$ The effect appears to be universal but there is still considerable uncertainty as to the mechanism responsible. We have previously studied OMR in OLED structures which used $N, N^{\prime}$-diphenyl- $N, N^{\prime}$ bis(3-methylphenyl)-(1, $1^{\prime}$-biphenyl)$4,4^{\prime}$ diamine (TPD) as the hole transport layer and $\mathrm{Alq}_{3}$ as the electron transport and emitting layer. These devices have been studied as a function of both cathode material ${ }^{7}$ and $\mathrm{Alq}_{3}$ thickness ${ }^{9}$ and we have found in all cases that the OMR was intimately linked to the onset of light emission from the device which suggested that excitons were responsible. We have recently investigated the OMR of these devices under illumination, ${ }^{10}$ which provides an external source of excitons, and found that under these conditions OMR could be observed below "turn on." On the basis of these results, we have suggested that the mechanism behind OMR is the interaction of free carriers with triplet excitons which act as scattering centers, lowering the mobility.

Some papers have commented that OMR can be observed in devices with unipolar transport. Francis et al., ${ }^{4}$ for example, studied OMR in PFO devices as a function of the cathode material and noted that OMR was observed in "hole

${ }^{a)}$ Electronic mail: w.gillin@gmul.ac.uk. only" devices with a gold cathode. In these devices, OMR was only observed at drive voltages $>40 \mathrm{~V}$ where the authors stated that "weak electroluminescence is observed" which suggests that despite the use of gold as a cathode, both electron injection and exciton formation were occurring and hence the material was supporting bipolar current transport. Recently, OMR has been observed in the highly doped conducting polymer poly $(3,4$-ethylenedioxythiophene) poly(styrenesulfonate) (PEDOT). In this work, ${ }^{11}$ indium tin oxide (ITO)/PEDOT/Ca devices demonstrated OMR at voltages as low as $0.2 \mathrm{~V}$ at room temperature. PEDOT is generally considered to be a hole transporting layer and little information is available on its electron transport properties. However, in this work, it is notable that OMR was only observed with a calcium cathode (not with aluminum or gold cathodes) and hence the injection process was non-Ohmic and the carrier concentrations far lower than with either $\mathrm{Al}$ or $\mathrm{Au}$ contacts. However, OMR was observed in both forward and reverse biases and this strongly suggests that the transport was unipolar. Nguyen et al. ${ }^{11}$ suggested that the conductivity in these samples, may be due to charges provided by the dopants rather than injected charge and OMR in PEDOT, requires further study.

In this work, we investigate the OMR of TPD, which is widely credited as being a hole transport material. We have found that for an ITO/TPD/Al device, OMR can be observed at voltages above $1.4 \mathrm{~V}$, which is the turn on voltage from the $I-V$ data. For devices with a thin layer of 2,9-dimethyl4,7-diphenyl-1,10-phenanthroline (BCP) to act as an electron injection/exciton blocking layer, we see OMR at voltages above $2.2 \mathrm{~V}$ but the shape of the OMR changes with drive voltage. Electroluminescence data from these devices show strong luminescence from the TPD which also shows that TPD can act as an electron transport material. The measured OMR is correlated with the light emission from the devices which is evident that it may be related to the presence of excitons.

\section{EXPERIMENTAL METHOD}

The basic device structure consists of an ITO coated glass substrate (purchased from Merck) with a sheet resistiv- 
ity of $\sim 13 \Omega / \square$ and $1000 \AA$ of TPD. On to these devices, a cathode was deposited consisting of $1000 \AA$ of aluminum. For devices with an electron injection layer/exciton blocking layer, a thin layer $(100 \AA$ or $200 \AA)$ of BCP was deposited onto the surface of the TPD followed by a $10 \AA$ layer of LiF before the aluminum evaporation. The TPD and BCP were purchased from Aldrich and purified using train sublimation prior to use. The ITO substrate was patterned using photolithography and cleaned by ultrasonicating in detergent solution, water, acetone, and chloroform. Following this, the ITO was treated in an oxygen plasma for $3 \mathrm{~min}$ at $30 \mathrm{~W}$ and 2.5 mbar pressure using a Diener Electronic Femto plasma system. The plasma treated substrate was immediately transferred to the deposition chamber for device fabrication. The deposition of the organic layers and metal electrodes were performed using a Lesker Spectros evaporation system with a base pressure during evaporation of $\sim 10^{-7}$ mbar. The rate of deposition of organic materials was about $2 \AA$ /s while that of the aluminum was varied from $\sim 1$ to $10 \AA$ /s. A calibrated oscillating quartz crystal monitor was used to determine the rate and thickness of the deposited layer. The whole device fabrication was performed without breaking vacuum.

Immediately after growth, the devices were placed in a light-tight sample holder with a calibrated silicon photodetector (Newport 818-SL) placed on the top surface of the device. The sample holder was placed between the poles of an electromagnet with the magnetic field perpendicular to the direction of current flow in the device. The photodetector was tested under various illumination levels to make sure there was no field dependence on its output. Measurements were taken with the device operated in constant voltage mode. Before and after each field measurement, a measurement at null field was taken. These two readings were averaged and used to determine the effect of the magnetic field. This procedure was performed in order to remove any effects due to drifting in the device characteristics. Voltage sourcing and current measurements were performed using a Keithley 236 source-measure unit with current measurements being averaged over 32 readings. The optical power output was measured using a Newport 1830 optical power meter.

Electroluminescence (EL) spectra of each device was recorded with the device being powered by a square wave and the EL dispersed in a Triax 550 spectrometer and detected using a photomultiplier and a Signal Recovery 7265 lock-in amplifier.

\section{RESULTS AND DISCUSSION}

Figure 1 shows the current density through the different devices, along with their emission intensity, as a function of drive voltage. For the device consisting $1000 \AA$ of TPD with an aluminum cathode, it can be seen that from the electrical characteristic, the device appears to switch on at $\sim 1.4 \mathrm{~V}$ although light output could not be observed in our measurement system until a drive voltage of $3.5 \mathrm{~V}$. Even here, the measured light output is very small and corresponds to a power efficiency of $\sim 10^{-7} \%$ which rises to a maximum of $\sim 10^{-6} \%$. The introduction of an electron injection/exciton blocking layer, along with a $\mathrm{LiF} / \mathrm{Al}$ cathode, increases the

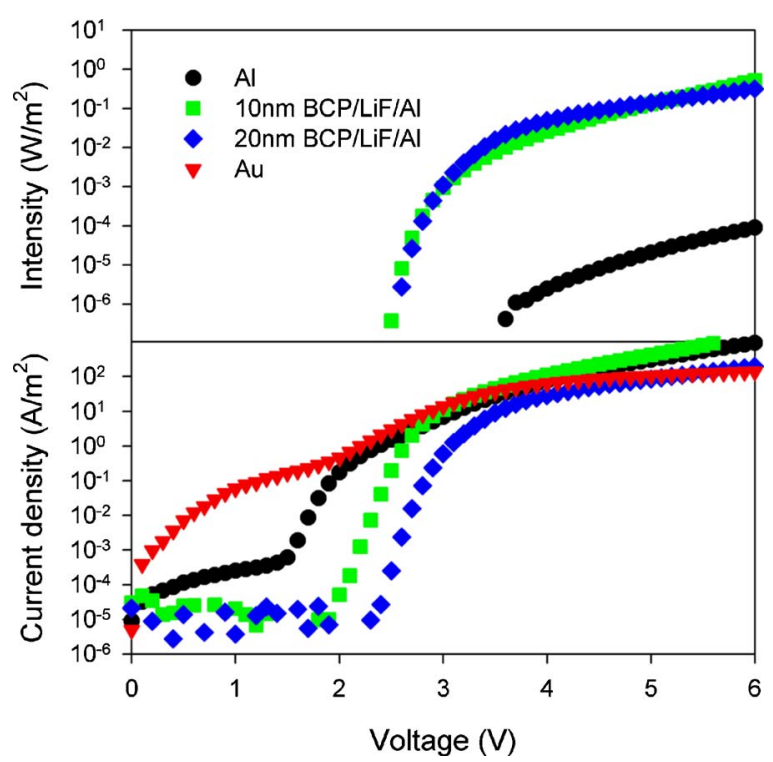

FIG. 1. (Color online) The current density and emission intensity as a function of drive voltage for the TPD based devices.

turn on voltage to $\sim 2 \mathrm{~V}$ for a $100 \AA$ BCP layer and $2.4 \mathrm{~V}$ for the $200 \AA$ BCP layer. The presence of these exciton blocking layers dramatically improves the efficiency of the devices with light becoming visible at $2.5 \mathrm{~V}$ for the $100 \AA$ $\mathrm{BCP}$ device and $2.6 \mathrm{~V}$ for the $200 \AA \mathrm{BCP}$ device. The measured efficiency for the $100 \AA$ BCP device was $\sim 10^{-4} \%$ at $2.4 \mathrm{~V}$ rising to a maximum of $\sim 10^{-2} \%$ while the maximum efficiency of the $200 \AA$ BCP device was $\sim 0.1 \%$.

Figure 2 shows the electroluminescence spectra from a "pure" TPD device and from the two devices with BCP injection layers. Also shown are the photoluminescence spectra from TPD and BCP. All of the TPD based devices show characteristic electroluminescence for the TPD layer. The peak at $422 \mathrm{~nm}$ seen in the TPD photoluminescence is visible for all devices but there is also considerable electrolu-

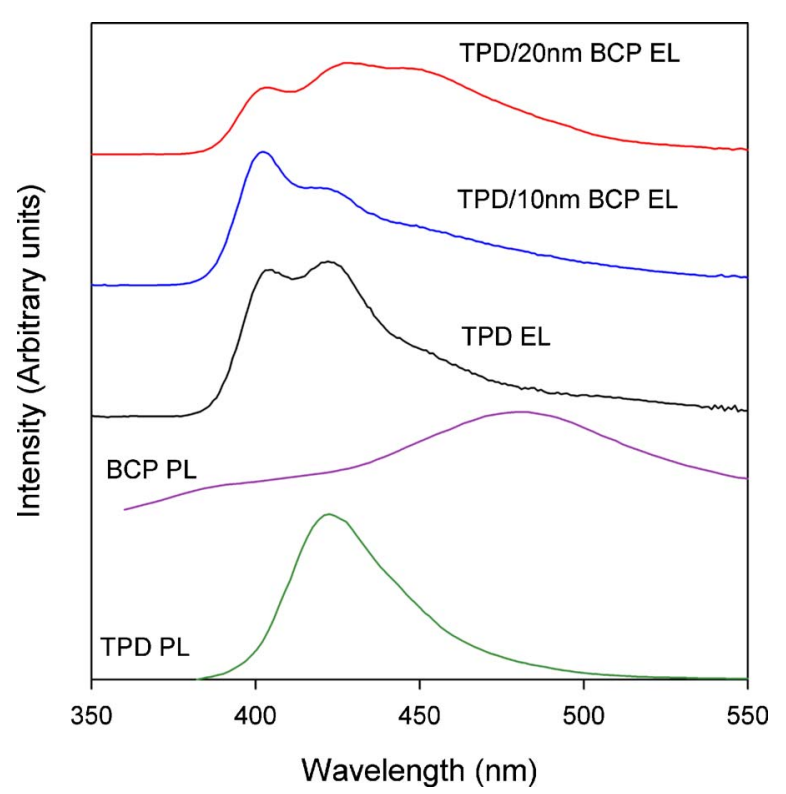

FIG. 2. (Color online) The photoluminescence spectra for TPD and BCP and the electroluminescence spectra for the corresponding devices. 


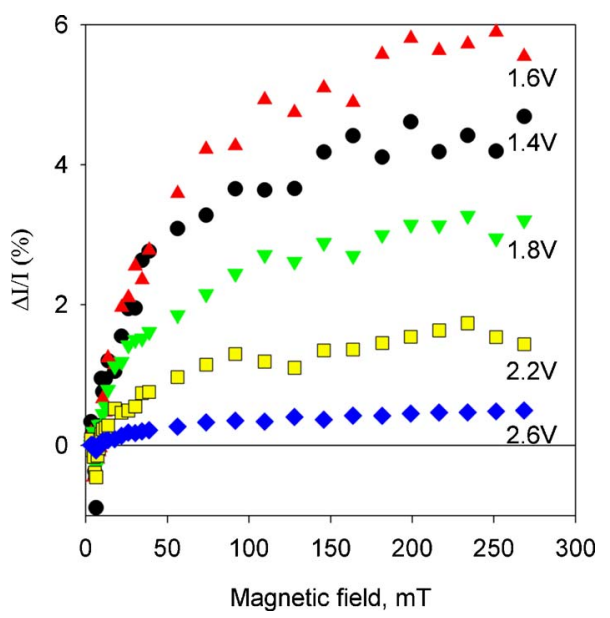

FIG. 3. (Color online) Magnetoresistance data as a function of magnetic field for an ITO/TPD/Al device structure at different drive voltages.

minescence from the other vibrational states. For the layers with BCP injection layers, there is no evidence of emission at wavelengths less than $390 \mathrm{~nm}$, where some BCP photoluminescence is visible, which suggests that the majority of the exciton recombination is occurring within the TPD. The fact that light emission is occurring from the TPD is a conclusive proof that the material is supporting both electron and hole transports. For the devices with BCP, the light output is highly correlated with the inflexion in the current density with applied voltage seen in Fig. 1. For the TPD only devices, this correlation is poor but this is due to the poor efficiency of these devices.

Figure 3 shows the magnetoresistance (percentage change in current) as a function of magnetic flux density for the ITO/TPD/Al device for voltages between 1.4 and $2.6 \mathrm{~V}$, below $1.4 \mathrm{~V}$, no magnetoresistance could be observed. From Fig. 1, it can be seen that $1.4 \mathrm{~V}$ corresponds to the point in the $J-V$ characteristic where the current through the device starts to rapidly increase. Although light output cannot be detected until $3.5 \mathrm{~V}$ due to the very low efficiency of this device structure, it appears that $1.4 \mathrm{~V}$ corresponds to the onset of electron injection. For the devices with the BCP injection/exciton blocking layer, the onset of magnetoresistance occurs at 2.2 and $2.4 \mathrm{~V}$ for the 100 and $200 \AA \mathrm{BCP}$ layers, respectively, and again these values correspond to the inflection points in their respective $J-V$ characteristics. For these two devices, light output could be detected at 2.5 and $2.6 \mathrm{~V}$ for the 100 and $200 \AA \mathrm{BCP}$ devices. For an ITO/ TPD/Au device over the same voltage range, no magnetoresistance was observed despite the current in the device at low voltages being greater than in the other devices. A weak OMR started to become visible for these devices at drive voltages $>7 \mathrm{~V}$ and weak electroluminescence could be observed at drive voltages $>12 \mathrm{~V}$. The presence of electroluminescence in the Au devices shows that electron injection is occurring from the Au cathode, although the efficiency of the electron injection is very low, and the current is dominated by hole transport. As a measure of the inefficiency of electron injection from the gold contact the power efficiency of this device at $15 \mathrm{~V}$ was only $10^{-11} \%$.

These results show that although OMR can be measured in "classic hole-transport" materials. The onset of OMR correlates strongly with the onset of light emission which implies that there must be excitons present and hence electron injection and transport. The result for the Au cathode device shows that at low voltages, where the current can be expected to be unipolar, no OMR was observed even though the current densities are up to five orders of magnitude greater than that where the onset of OMR is observed with other cathodes. Again, this suggests that current density alone is not responsible for OMR but that the presence of excitons is essential.

For the ITO/TPD/Al device, the shape of the magnetoresistance is dependent on the current density in the device. In Fig. 3, it can be seen that from the onset of OMR at $1.4 \mathrm{~V}$, the magnitude of the OMR increases at $1.6 \mathrm{~V}$ before steadily decreasing as the applied voltage increases towards $2.6 \mathrm{~V}$. The OMR also shows an interesting behavior at low fields. The OMR rapidly goes negative as the field is applied reaching a maximum negative value at $\sim 6 \mathrm{mT}$ before becoming positive again by $10 \mathrm{mT}$ and then increasing in magnitude. We have seen similar behavior in $\mathrm{Alq}_{3}$ based devices where the thickness of the $\mathrm{Alq}_{3}$ layer was very thin. ${ }^{9}$ In those devices, a large negative OMR was observed at low drive voltages and this was attributed to the dissociation of triplets at the cathode. As the drive current is increased, this process became dominated by a positive OMR which was tentatively attributed to the field dependence of the interaction of free carriers with triplet excitons. These two processes had different field dependences and at high current density, where the triplet-carrier interaction dominated, the OMR showed a small negative going spike, due to the dissociation current, followed by the positive OMR due to the carrier-triplet interaction. The change in the efficiency and electroluminescence intensity for this device with applied field is shown in Fig. 4. In $\mathrm{Alq}_{3}$ based OLEDs where, irrespective of device thickness or drive voltage, the efficiency always showed a saturation in the light output with increasing field which is a characteristic of a hyperfine scale dependence. This saturation in $\Delta \eta / \eta$ was always positive and independent of the sign of the change in current through the device. For the TPD based device, the efficiency was again found to be always positive but it did not saturate with increasing field. There is an initial rapid increase in efficiency at low field, which is typical of a hyperfine scale interaction, but at higher field, this is replaced by a second effect which is approximately linear with applied field. For this device, it can be seen that, within experimental error, the $\Delta \eta / \eta$ at the different drive voltages all appear to be identical. In the $\Delta \mathrm{EL} / \mathrm{EL}$ plot, however, it can be seen that the magnitude of $\Delta E L / E L$ appears to scale with drive voltage at high $\mathrm{B}$. This suggests that the increase in light output at high B is directly related to the increase in current in the device caused by the magnetic field. More work will need to be performed to understand what is responsible for this process.

The introduction of a layer of BCP between the TPD and the cathode dramatically affects the observed magnetoresistance. Figure 5 shows the OMR for the device with $100 \AA$ of BCP and the results for the $200 \AA$ BCP layer are qualitatively very similar. When the device first switches on (at 

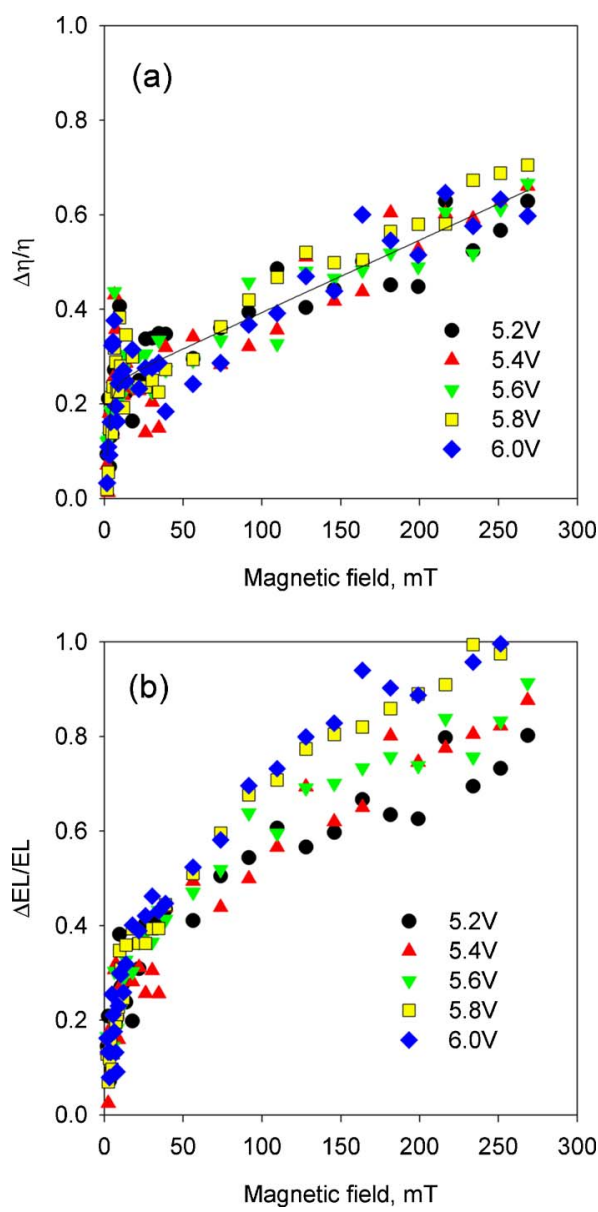

FIG. 4. (Color online) The change in efficiency for the ITO/TPD/Al device as a function of magnetic field (a) and the corresponding data for the electroluminescence output (b).

$2.2 \mathrm{~V}$ ) a negative OMR can be observed. This OMR has the characteristic shape due to hyperfine scale interactions and saturates as the field increases. As the applied voltage is increased, the magnitude of the OMR decreases, and at $\sim 4 \mathrm{~V}$, the OMR at high magnetic field strength becomes positive. At a drive voltage of $5.4 \mathrm{~V}$, there is a positive OMR, at high field, of $\sim 0.4 \%$ whereas at low field $(\sim 6 \mathrm{mT})$ the OMR is negative $(\sim-0.1 \%)$. This is identical to the behavior observed for the TPD only devices.

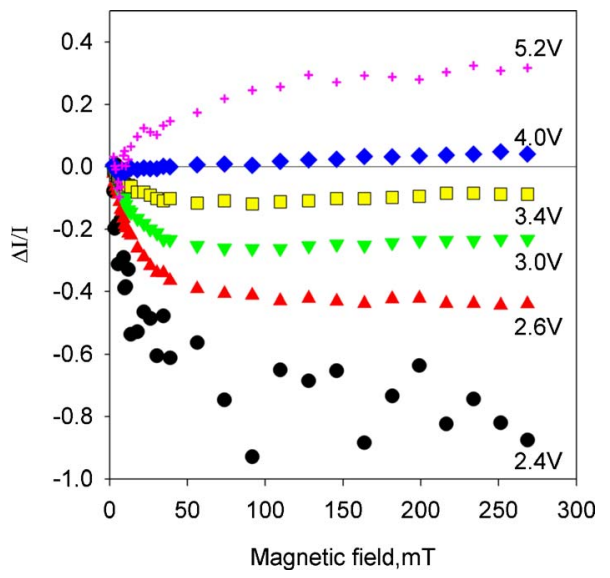

FIG. 5. (Color online) Magnetoresistance data as a function of magnetic field for an $\mathrm{ITO} / \mathrm{TPD} /(10 \mathrm{~nm}) \mathrm{BCP} / \mathrm{LiF} / \mathrm{Al}$ device structure at different drive voltages.

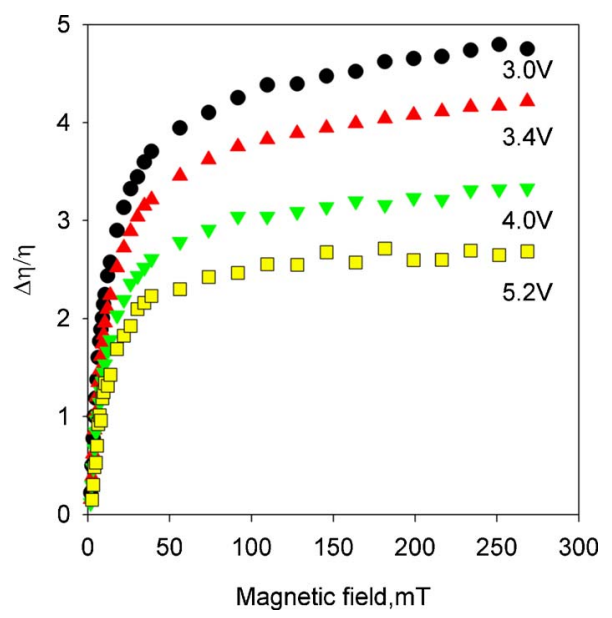

FIG. 6. (Color online) The change in efficiency for the ITO/TPD/ $(10 \mathrm{~nm}) \mathrm{BCP} / \mathrm{LiF} / \mathrm{Al}$ device structure as a function of magnetic field.

For the TPD only devices excitons could be dissociating at both the anode and cathode and hence the presence of the BCP between the TPD and the cathode will only stop dissociation at one of the interfaces. However, the presence of the BCP layer does effectively block hole transport directly to the cathode and this can be observed in the greatly reduced leakage currents observed in the devices with the BCP layer (Fig. 1). It is therefore possible that the negative OMR at low voltages seen in the TPD/BCP devices could be occurring in the TPD only devices but is being masked because this dissociation current has a negligible contribution to the overall current in the device at these low voltages (probably dominated by hole transport). It is only when the hole current is reduced and electron injection efficiency improved, through the addition of the BCP layer, that the contribution of the dissociation current becomes significant.

Figure 6 shows the change in efficiency of the $100 \AA$ $\mathrm{BCP} / \mathrm{TPD}$ as a function of magnetic field. It can be seen that although the OMR for this device is approximately zero at drive voltages around $4 \mathrm{~V}$, the efficiency is showing a change of up to $3 \%$ at this voltage and that the shape of the efficiency curves is identical as the drive voltage increases. This observation suggests that the change in current induced by the magnetic field is not producing a change in the carrier density that is responsible for the modulation in the light output as the magnetic field is applied.

\section{CONCLUSIONS}

These results show that the classic hole-transport material, TPD, can also support electron transport and that organic magnetoresistance can be observed in this material only at voltages above that required to obtain electron injection. In addition, it was observed that OMR cannot be seen in the unipolar transport region despite the current density, and hence carrier density, being orders of magnitude greater that that where OMR can be observed in a bipolar carrier regime. These observations supports the idea that excitons are a prerequisite for observing OMR in these organic materials. 
${ }^{1}$ J. Kalinowski, M. Cocchi, D. Virgili, P. Di Marco, and V. Fattori, Chem. Phys. Lett. 380, 710 (2003).

${ }^{2}$ R. P. Groff, R. E. Merrifield, A. Suna, and P. Avakian, Phys. Rev. Lett. 29, 429 (1972).

${ }^{3}$ R. P. Groff, A. Suna, P. Avakian, and R. E. Merrifield, Phys. Rev. B 9, 2655 (1974).

${ }^{4}$ T. L. Francis, Ö. Mermer, G. Veeraraghavan, and M. Wohlgenannt, New J. Phys. 6, 185 (2004).

${ }^{5}$ Ö. Mermer, G. Veeraraghavan, T. L. Francis, Y. Sheng, D. T. Nguyen, M. Wohlgenannt, A. Köhler, M. K. Al-Suti, and M. S. Khan, Phys. Rev. B 72, 205202 (2005).
${ }^{6}$ V. N. Priodin, J. D. Bergeson, D. M. Lincln, and A. J. Epstein, Synth. Met. 156, 757 (2006).

${ }^{7}$ P. Desai, P. Shakya, T. Kreouzis, W. P. Gillin, N. A. Morley, and M. R. J. Gibbs, Phys. Rev. B 75, 094423 (2007).

${ }^{8}$ Y. Wu, Z. Xu., B. Hu, and J. Howe, Phys. Rev. B 75, 035214 (2007).

${ }^{9}$ P. Desai, P. Shakya, T. Kreouzis, and W. P. Gillin, J. Appl. Phys. 102, 073710 (2007).

${ }^{10}$ P. Desai, P. Shakya, T. Kreouzis, and W. P. Gillin, Phys. Rev. B 76, 235202 (2007).

${ }^{11}$ T. D. Nguyen, Y. Sheng, J. Rybicki, G. Veeraraghavan, and M. Wohlgenannt, J. Math. Chem. 17, 1995 (2007). 